\title{
Distributed System SCADA using Multiple Industrial Communication Protocols on Allen Bradley Equipment
}

\author{
Wilson Sánchez Ocaña ${ }^{1 *}$, Óscar Proaño², Pablo Salazar², Elizabeth Salazar', Washington Loza' and \\ Juan Esteban Sánchez ${ }^{3}$ \\ 'Departamento de Eléctrica y Electrónica, Universidad de las Fuerzas Armadas ESPE, ID: 60104598, Sangolquí, \\ Ecuador;wesanchez@espe.edu.ec,netzonews@hotmail.com,w_loza@hotmail.com \\ 2Departamento de Energía y Mecánica, Universidad de las Fuerzas Armadas ESPE, ID: 60104598, Sangolquí, \\ Ecuador; ppsalazar@espe.edu.ec \\ 3Facultad de Ciencias de la Salud, Universidad de las Américas, Quito, Ecuador; juan.sanchez.salazar@udla.edu.ec
}

\begin{abstract}
Background/Objectives: This research is aimed at designing and implementing a SCADA system with multiple industrial communication protocols, tuning of composite PID controls in multivariable processes and configuration of industrial networks in high-end Allen Bradley equipment. Methods: The SCADA behavior was identified according to the variation of parameters within the system, and the responses obtained through a datalogg, as well as the validation of the operation of the PID control according to the trend of the curves generated based on the controlled processes through the display of the View Panel in case of operators or an application for monitoring and control carried out in Factory Talk Site Edition, in addition, the reliability of the industrial networks was verified through the diagnosis of each protocol. Findings: Findings: With the SCADA system, and the use of multiple communication protocols, the sending and reception of data generated in the field devices was managed, and the trends and behavior of the physical variables of the process were obtained in real time, as well as a database for the analysis, diagnosis and validation of the functionality of the distributed processes, both at the liquid level and at the speed control level; in addition, the stability of the Industrial Ethernet (EI), ControlNet and DeviceNet industrial communication networks was verified in the face of disturbances and changes in the state of the industrial processes. Applications/Improvements: Database generation, variable status identification, historical data and alarms; to improve the effectiveness of your systems, reduce the costs of predictive and preventive maintenance, operation and production of different industrial processes.
\end{abstract}

Keywords: ControlNet, DeviceNet, Ethernet Industrial, Factory Talk, Scada

\section{Introduction}

From the first automated machine based on electromechanical components to large installations made up of a multitude of machines working in coordination, there has been a common denominator that has always been present to a greater or lesser extent: the relationship between the machine and its environment.
A company needs to incorporate an integrated communications system in order to obtain a benefit, such as: reduction of production costs, improvement of quality, improvement of productivity, reduction of storage or inventory, improvement of the effectiveness of its systems, reduction of maintenance costs ${ }^{1}$.

Modern industries have systems or equipment that work at different levels of automation, but at the same

${ }^{*}$ Author for correspondence 
time they need to be linked together to work in an integrated manner and thus obtain a satisfactory joint result, which depends on communication from the control room to the field instruments, thus forming the pyramid of CIM automation?

Figure 1 shows the pyramid of automation with the equipment that was used in the development of the research.

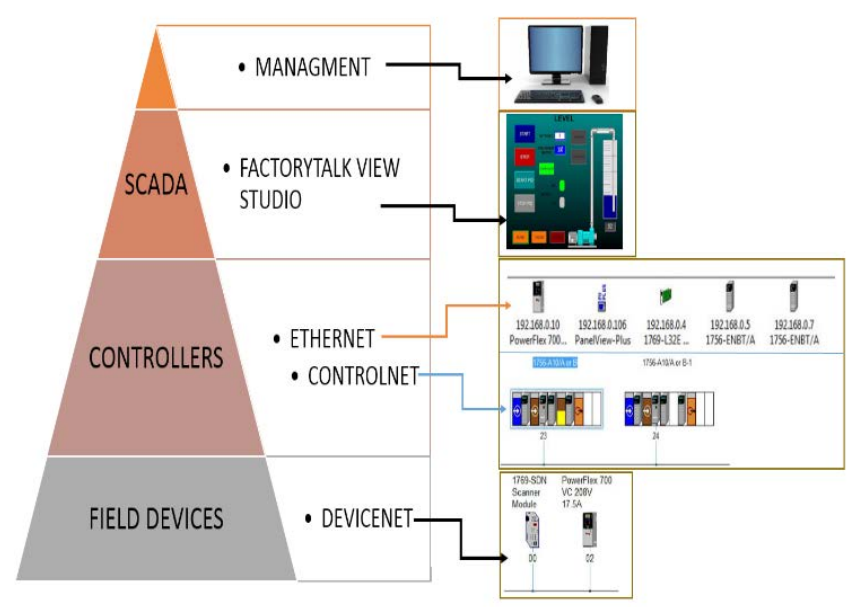

Figure 1. Pyramid of automation with Allen-Bradley equipment.

Communication protocols have evolved rapidly as technology has advanced, mainly in terms of controllers, which, through their use, allow several stages of a process to be linked together in isolation ${ }^{2}$.

The industrial communication protocols also have advantages such as:

- Greater measurement accuracy

- Increased and better availability of information

The DeviceNet communication protocol fulfills the objective of being able to connect industrial devices to automate any process where communication can be carried out between controllers, sensors or actuators, as is the case of the present project where DeviceNet communication is controlled between a high-end modular PLC Compact_Logix E32 with SDN communication card, a frequency converter Power Flex 700, with the speed control process with a three-phase induction motor ${ }^{3}$.

Working with a ControlNet, which is an open protocol network and also meets a variety of applications based on high real-time performance and high speed, this type of network allows real-time interlocking of I/O control- lers, drives and valves. As can be seen in this project, the control of 2 PLC's Control_Logix L61 will be executed, with a CNB communication card, a Power Flex 700 frequency converter, with the process of liquid level control with a three-phase induction motor ${ }^{4}$.

Figure 2 shows the industrial networks that are implemented in research:

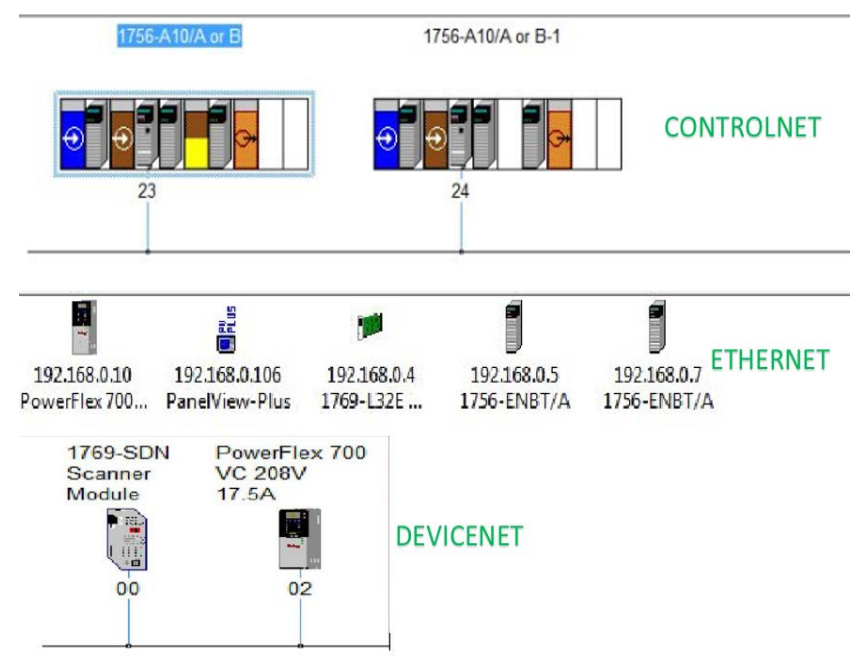

Figure 2. Industrial networks EI, ControlNet, DeviceNet.

By means of Factory Talk View Machine Edition (ME), Figure 3, an HMI can be made with a Panel View Plus 1000, which provides the solution dedicated to the generation of a Man-Machine interface, considering that it provides information on the process, monitoring and control of physical variables, curve detail and generation of history and alarms ${ }^{\underline{5}}$.

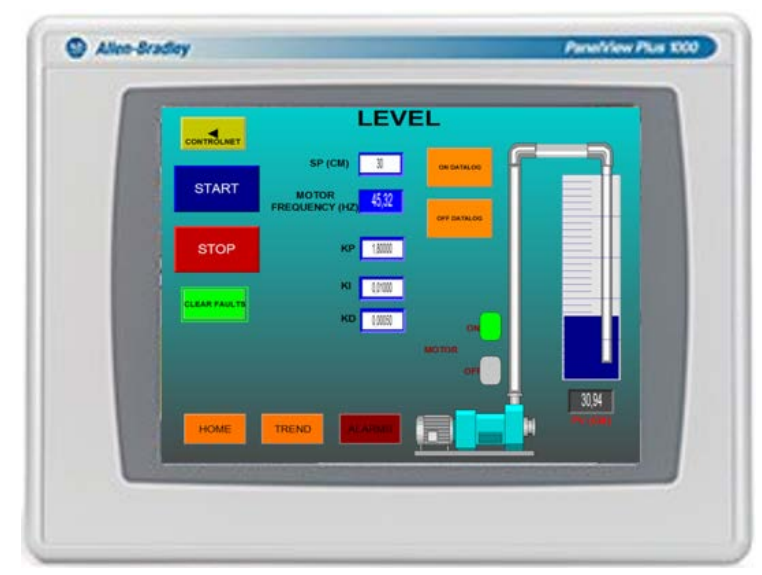

Figure 3. FactoryTalk View ME.

The Factory Talk Site Edition (SE) Figure 4, is a system that allows the supervision and control of processes 
that are of individual lines taking into account that it is possible to execute the activities according to an operator, data service, clients in different PC's allowing this way an independent work ${ }^{6}$.

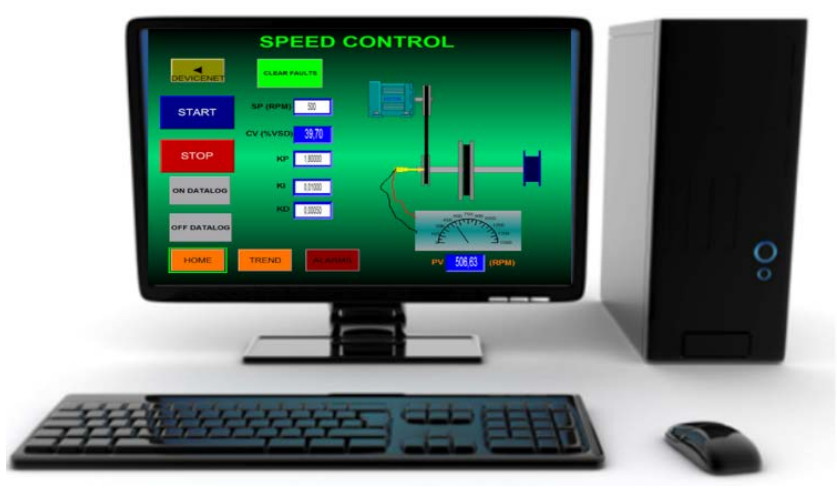

Figure 4. FactoryTalk View SE.

\section{Materials and Methods}

This experiment involved the creation of a SCADA based on the pyramid of automation generated with Allen Bradley equipment. The research includes multiple industrial networks, the EI (Industrial Ethernet) used in SCADA, the ControlNet network to communicate highend controllers, and DeviceNet as a communication network between controllers and field devices. For the implementation of the DeviceNet network, a Compact Logix $1769 \mathrm{~L} 32-\mathrm{E}^{7}$ PLC was used, the device is connected to an Ethernet network via its CPU, the connection through this network has a DeviceNet 1769-SDN module, which allows communication with any device that has this type of protocol, the main module acts as the master and each of the nodes as a remote slave or chassis $^{\underline{8}}$, in one node of this network is connected the Power Flex 700 variable speed drive that allows the control of three-phase induction motors in simple and complex controls used in pumping, speed control and positioning systems ${ }^{9}$; will control the speed generated in the threephase Siemens 1LA7 12-2YA60 motor of 5HP where its rotation is sensed by a generator block that emits a voltage signal $(0 \ldots 10) \mathrm{Vdc}$ and is received as an analog signal by the PLC in order to carry out the PID control based on the programming and tuning made in the RsLogix 5000, software that allows configuration, programming and interconnecting the high-end Allen Bradley products with each other ${ }^{10}$, considering that for the interconnection between devices with DeviceNet technology it is necessary to use the RsNetwork for DeviceNet, a computer tool that allows you to develop the configuration of the devices, in this case between the Power Flex Inverter and the PLC $\stackrel{11}{ }$.

For the ControlNet network, a coaxial cable with RG-6 ${ }^{12}$ quad shielding is used to transmit information between the Control Logix 1756-L61 controllers, which is defined as a scalable controller in which several $\mathrm{I} / \mathrm{O}$ points can be incorporated, and for this communication a Control Net 1756 -CNB/ Revision 15 module is required to carry out the Control Logix applications $\frac{13}{}$, one controller is declared as a slave and the other as a master and the other is generated.

communication for data transfer, or under consideration of main PLC and remote chassis; receiving the plant information through the level sensor in the main PLC analog input module of node 23 and the response of the process output through the remote chassis node 24 , for which the use of RsNetwork for ControlNet is required, where the network configuration is made graphically of all the nodes with a scanner and automatic mapping and with the respective network data ${ }^{14}$.

For the development of the PID control of the level variables of one plant and the speed of the motor of the other plant, a manual tuning is made, configuring each one of the constants of the controller, these are Kp, Ki and $\mathrm{Kd}$. After obtaining the values of the constants, the values are set in the PID block of the ladder programming in RSLogix 5000. Figure 5 shows the programming of the composite PID control of the process controllers.

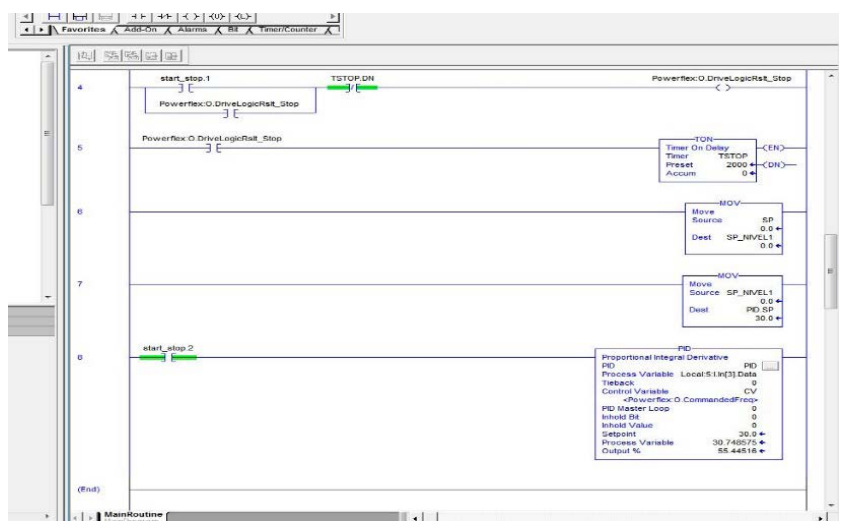

Figure 5. Programming the PID composite control.

A View Plus 1000 Panel was used for the HMI, connected to the EI network ${ }^{15}$. The computer platform used for the HMI is Factory Talk ME, and for the Factory Talk Site Edition (SE) SCADA system, Figure 6 shows the HMI 
for the level control process and Figure 7 shows the HMI for the engine speed control.

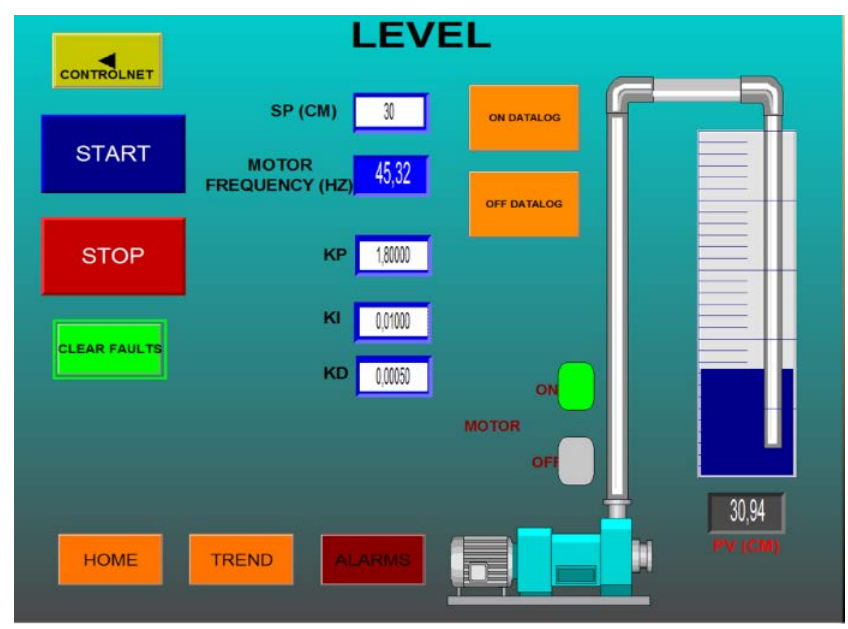

Figure 6. Scada - HMI of the level process.

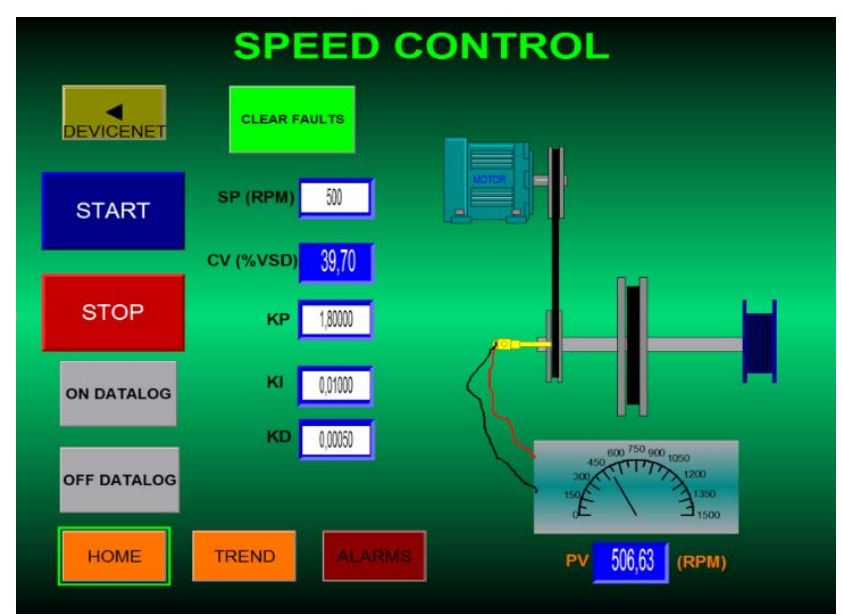

Figure 7. Scada -HMI of the speed control process.

\section{Result and Discussion}

The SCADA system allows the interaction of the physical process with the virtual one in terms of both level and speed, as well as an alarm report to independently identify the steps that are being carried out either by the operator or by the system itself, that is to say, if it should present any type of inconvenience in its operation according to the pre-established.

The communication between the industrial communication protocols Control Net and DeviceNet, allows a simultaneous and error-free work according to both physical and virtual operation, taking into account that they are two completely different processes but can be controlled from the same device, where if one of these reaches a failure or malfunction would not imply any inconvenience with the other.

With the network topology defined in Figure 2 of the ControlNet and DeviceNet networks, the diagram of how they are connected to each of their devices within the network is defined. Additionally, for the SCADA system and process control via the Panel View Plus 1000, an Industrial Ethernet network and remote control in Factory Talk SE were used, completing the communication pyramid with the Allen-Bradley equipment.

Considering that the control of the processes is done purely by the IPR ports of the field devices (Power Flex 700 ), while on the other hand the use of analog modules is only necessary for the data acquisition of the sensors (both level and speed), the PID control constants adjustment and the Set-Point input can be done from the Factory Talk SE client while from the HMI only the Set-Point data can be entered and the composite control constants, the indicators of each of the variables that allow us to visualize the values that are generated both in level and speed, are shown both in the HMI for the operator and in the computer through the FT SE client; Figures 8 and 9 show the Process Variables (PV), Controlled Variables (CV) and Set-Point (SP) as a function of both speed and level control time.

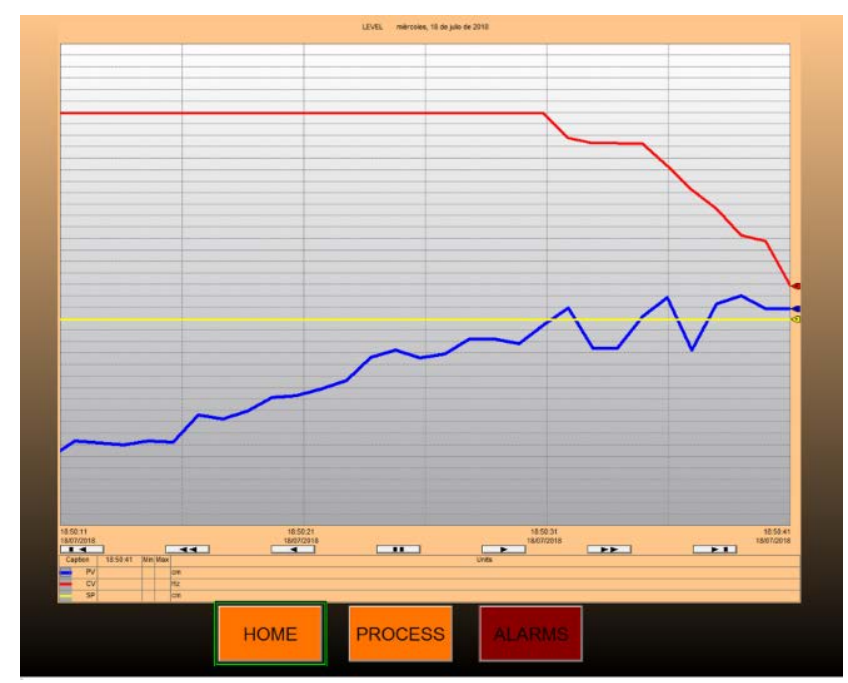

Figure 8. Level control variables graph.

It is also essential to bear in mind that the SCADA is carried out on a PC, where additionally, Figure 10 shows a file of data generated in the DATALOG. 


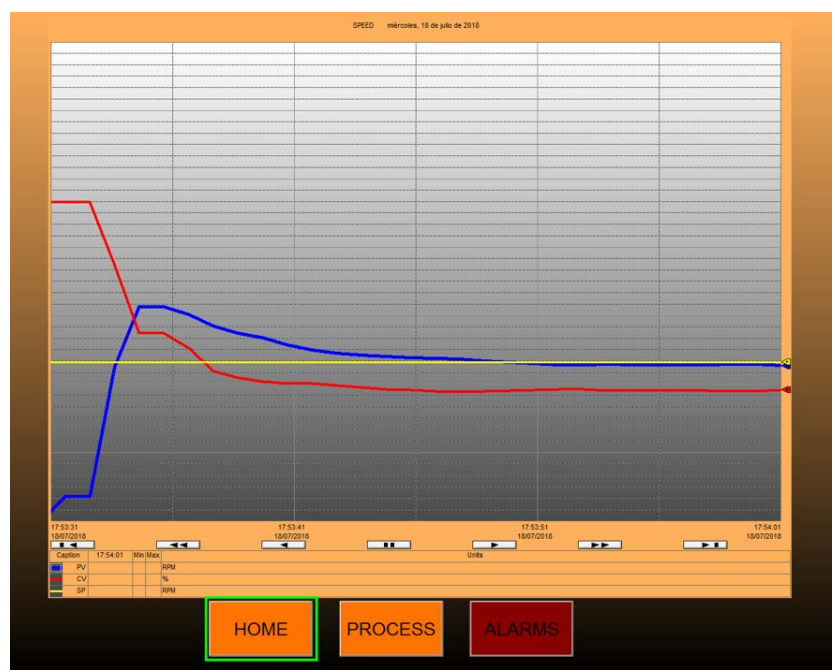

Figure 9. Speed control variables graph.

\begin{tabular}{|c|c|c|c|}
\hline Nombre & Fecha de modifica... & Tipo & Tamaño \\
\hline 201803150000 (Float).DAT & 15/03/2018 10:00 & Archivo DAT & $2 \mathrm{~KB}$ \\
\hline 201803150000 (String).DAT & 15/03/2018 9:58 & Archivo DAT & $1 \mathrm{~KB}$ \\
\hline 201803150000 (Tagname).DAT & 15/03/2018 9:58 & Archivo DAT & $1 \mathrm{~KB}$ \\
\hline 201803290000 (Float).DAT & $29 / 03 / 201813: 18$ & Archivo DAT & $2 \mathrm{~KB}$ \\
\hline 201803290000 (String).DAT & 29/03/2018 13:16 & Archivo DAT & $1 \mathrm{~KB}$ \\
\hline 201803290000 (Tagname).DAT & 29/03/2018 13:16 & Archivo DAT & $1 \mathrm{~KB}$ \\
\hline 201804030000 (Float).DAT & 03/04/2018 16:34 & Archivo DAT & $22 \mathrm{~KB}$ \\
\hline 201804030000 (String).DAT & 03/04/2018 16:04 & Archivo DAT & $1 \mathrm{~KB}$ \\
\hline [ 201804030000 (Tagname).DAT & 03/04/2018 16:04 & Archivo DAT & $1 \mathrm{~KB}$ \\
\hline DATABASE1.DLG & 03/04/201816:04 & Archivo DLG & $1 \mathrm{~KB}$ \\
\hline DATABASE1.DNS & 03/04/2018 16:34 & Archivo DNS & $1 \mathrm{~KB}$ \\
\hline
\end{tabular}

Figure 10. Files generated in DATTALOG.

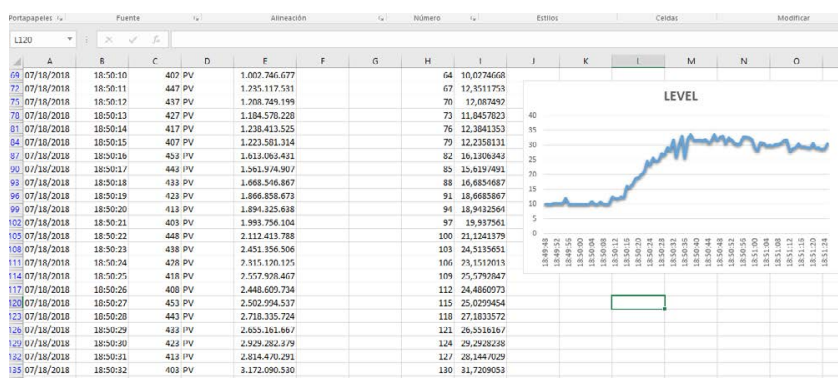

Figure 11. Level process Datalog Grap.

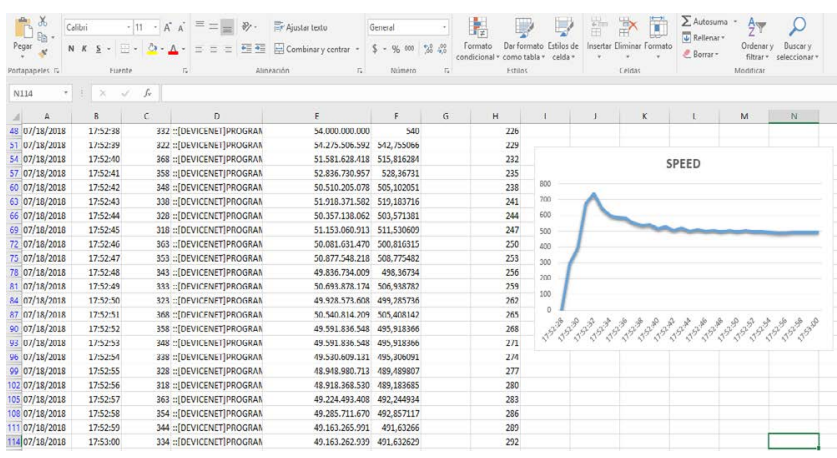

Figure 12. Speed process Data Log Graph.
If we graph the data generated in the DATALOG, the variables PV and SP, gives as a result the graph of Figure 11, similar to the one generated in the level SCADA system, with an acceptable error due to the number of samples taken. Similarly, Figure 12 was generated, similar to the one generated in the speed SCADA system, highlighting the importance of the graphs observed in real time and those generated from the databases.

\section{Conclusion}

Data acquisition, historical and alarm generation, optimization of communication networks from Industrial Ethernet for the SCADA system, ControlNet for controllers and DeviceNet for controllers and field devices, from a supervision and monitoring system both local and remote, ensure the reliability and efficiency of the operation of distributed systems.

The visualization of data in the HMI in real time is of great importance since the process indicators are shown and its correct operation is verified. On the other hand, the generation of historical data through the DATALOG is very important to obtain trend graphs, to make an analysis and diagnosis of the processes in order to make decisions based on real events.

\section{References}

1. Sánchez OW, Proa-o GO, Saigua PJ, Pozo JJ, Terán HH, y Silva MM. Scada of distributed systems through multiple industrial communication protocols. International Journal of Pure and Applied Mathematics. 2018 Apr; 119(15):295762.

2. Castro M, Díaz G, Mur F, y Fernández R. Principios Básicos de Comunicaciones Industriales, Madrid, Librería UNED; 2007.

3. Biegacki S, y VanGompel D. The application of DeviceNet in process control. ISA Transactions. 1996; 35:169-76. https://doi.org/10.1016/0019-0578(96)00022-5

4. Rockwell Automation. ControlNet- Network [Internet]. [cited 2018 Apr 18]. Available from: https:// ab. rockwellautomation. com/ Networks -and- Communications/ ControlNet- Network.

5. RockwellAutomation. Factorytalk View me [Internet]. [cited 2018 Apr 18]. Available from: https://www. rockwellautomation.com/rockwellsoftware/products/factorytalk-view-me.page.

6. Lema JC, Sánchez R, y Escudero M. Módulo didáctico para control y monitoreo de 3 variables nivel, temperatura, pre- 
sión, implementado con sensors. PLC Flexlogix y SCADA Factory Talk (Allen Bradley) [Internet]. [cited 2018 Apr 18]. Available from: https://www.dspace.ups.edu.ec/bitstream/123456789/4663/1/UPS-GT000416.pdf.

7. Bradley A. Manual del usuario de los controladores CompactLogix 1769 [Internet]. [cited 2018 Apr 18]. Available from: http://literature.rockwellautomation.com/ idc/groups/literature/documents/um/1769-um011_-es-p. pdf.

8. Bradley A. Compact TM I/O 1769-SDN DeviceNet Scanner Module [Internet]. [cited 2018 Apr 18]. Available from: http://docs-asia.electrocomponents.com/ webdocs/0648/0900766b806488fb.pdf.

9. Bradley A. Variadores de CA PowerFlex 700 [Internet]. [cited 2018 Apr 18]. Available from: https:// ab.rockwellautomation.com/es/Drives/PowerFlex-700.

10. Rockwell Automation. Studio 5000 Logix designer [Internet]. [cited 2018 Apr 17]. Available from: https:// www.rockwellautomation.com/rockwellsoftware/products/ studio5000-logix-designer.page.
11. Bradley A. Getting results with RSNetWorx for DeviceNet [Internet]. [cited 2018 Apr 17]. http://literature.rockwellautomation.com/idc/groups/literature/documents/gr/ dnet-gr001_-en-e.pdf.

12. Bradley A. Rockwellautomation. ControlNetNetwork [Internet]. [cited 2018 Apr 17]. https:// ab.rockwellautomation.com/ es/ Networks- andCommunications/ ControlNet-Network.

13. Rockwell Automation. ControlLogix ControlNet Interface Module, Series E [Internet]. [cited 2018 Apr 17]. http:// literature.rockwellautomation.com/idc/groups/literature/ documents/rn/1756-rn627_-en-p.pdf.

14. Rockwell Automation. Getting Results with RSNetWorx for ControlNet [Internet]. [cited 2018 Apr 17]. http://literature.rockwellautomation.com/idc/groups/literature/ documents/gr/cnet-gr001_-en-e.pdf.

15. Rockwell Automation, Terminales PanelView 1000 [Internet]. [cited 2018 Apr 17]. http://literature.rockwellautomation.com/idc/groups/literature/documents/ in/2711-in036_-es-e.pdf. 